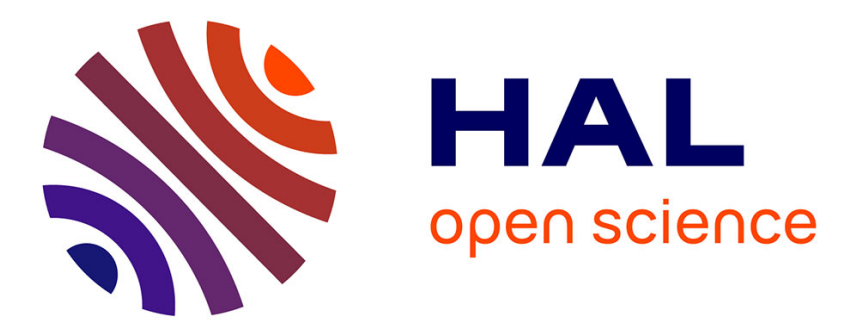

\title{
La fixation anale dans la paranoïa. Un cas clinique exemplaire et ses prolongements théoriques
}

Benjamin Levy, Cécile Prudent, Renaud Evrard, Christophe Clesse, Michel Decker, Claude de Tychey

\section{- To cite this version:}

Benjamin Levy, Cécile Prudent, Renaud Evrard, Christophe Clesse, Michel Decker, et al.. La fixation anale dans la paranoïa. Un cas clinique exemplaire et ses prolongements théoriques. L'Évolution Psychiatrique, 2018, 83 (2), pp.287-303. 10.1016/j.evopsy.2017.06.002 . halshs-02137012

\section{HAL Id: halshs-02137012 \\ https://shs.hal.science/halshs-02137012}

Submitted on 22 May 2019

HAL is a multi-disciplinary open access archive for the deposit and dissemination of scientific research documents, whether they are published or not. The documents may come from teaching and research institutions in France or abroad, or from public or private research centers.
L'archive ouverte pluridisciplinaire HAL, est destinée au dépôt et à la diffusion de documents scientifiques de niveau recherche, publiés ou non, émanant des établissements d'enseignement et de recherche français ou étrangers, des laboratoires publics ou privés. 
Lévy, B., Prudent, C., Evrard, R., Decker, M., Clesse, C., De Tychey, C. (2017). La fixation anale dans la paranoïa. Un cas clinique exemplaire et ses prolongements théoriques. L'Evolution psychiatrique. doi: 10.1016/j.evopsy.2017.06.002.

Résumé : Cet article présente la spécificité de la fixation anale dans le délire paranoïaque (délire de persécution). Les auteurs s'appuient sur un cas clinique ayant valeur d'exemplarité afin d'en saisir la portée, la place et le rôle. Le protocole Rorschach du sujet est étudié dans cette visée. Il est resitué dans le contexte d'une recherche quantitative et comparative en cours portant sur l'approche clinique projective différentielle des organisations psychotiques dissociées.

Mots-clés : paranoïa, psychoses, test Rorschach, érotisme anal

Abstract : This paper presents the distinctive features of anal fixation in paranoid delusion (delusion of persection). The auteurs base their hypothesis on the exemplary nature of a clinical case so as to better understand its scope, its place and its function. Rorschach protocol is examined into this view. It is resituated into the contexte of a current quantitative and comparative research on projective-test based differential approach of dissociative psychotic organisations.

Keywords : paranoia, psychosis, Rorschach test, anal eroticism

\section{La fixation anale dans la paranoïa}

\section{INTRODUCTION}

Actuellement, la paranoïa pose, sur le plan théorico-clinique, des problèmes de fond au niveau de son statut diagnostique en psychiatrie [1] puisqu'elle a disparu de certains modèles alors qu'elle conserve toute sa place en tant qu'entité psychotique dans certaines nosographies psychodynamiques françaises et américaines (Bergeret, 1974 ; Mc Williams et al., 2013). Que doit-on faire des signes cliniques relatifs à la paranoïa, héritages d'une tradition clinique plus que centenaire ? Ont-ils également perdu de leur tranchant et de leur pertinence?

Dans cet article, nous nous intéresserons à la place et au rôle de l'érotisme anal dans le délire paranoïaque. Dans un premier temps, nous emprunterons une perspective historique en revenant sur les élaborations théoriques de Freud, dans son travail consacré aux Mémoires du Président Schreber [1911c], mais aussi de ses successeurs, tels qu'Eduard Hitschsmann (1871-1957), Johannes Ophuijsen (1882-1950) ou August Stärke (1880-1954).

Dans un deuxième temps, nous développerons une perspective clinique autour du cas de Manuel, aujourd'hui âgé de 33 ans, suivi en psychiatrie depuis deux ans suite au développement d'un délire paranoïaque, prenant pour origine la conviction d'avoir fait l'objet d'une effraction anale durant son sommeil. Une hésitation diagnostique sourde à l'abord d'un tel tableau : au sens du DSM-5 [4], ce cas témoigne d'une co-morbidité entre les troubles de la personnalité paranoïde («Paranoid Personality Disorder») et les troubles délirants («Delirium Disorder»); tandis que, selon les critères diagnostiques relatifs au 
modèle de référence psychanalytique qui est le nôtre, tous les éléments cliniques de la paranoïa y semblent réunis.

Le cas de Manuel aura d'une part valeur d'exemplarité et viendra d'autre part s'appuyer sur des données quantitatives issues d'une recherche comparative en psychiatrie à travers le test de Rorschach. Nous nous demanderons, lors d'un troisième temps, en quoi les épreuves projectives telles que le Rorschach sont susceptibles de mettre en évidence l'érotisme anal en tant que signe clinique.

Enfin, dans une dernière partie, nous reviendrons sur les travaux essentiels de Karl Abraham (1877-1925), démontrant comment l'étude de thématiques liées à l'analité permet de proposer un diagnostic différentiel entre schizophrénie, schizophrénie paranoïde et paranoïa.

Dans son ensemble, cet article se propose donc de présenter une synthèse détaillée sur la valence anale, considérée comme l'une des thématiques de prédilection dans le délire paranoïaque, et de questionner plus généralement la clinique des psychoses.

\section{LA THEMATIQUE DE L'EROTISME ANAL DANS LA PARANOÏA : APPORTS DE SIGMUND FREUD ET DE SES CONTEMPORAINS}

\section{Freud, le Président Schreber et les Mémoires d'un névropathe}

Pour commencer à jeter quelque lumière sur les convictions délirantes qui, chez Manuel, touchent aux problématiques anales, nous souhaitons rappeler certains pans de la théorisation psychanalytique concernant ce sujet précis.

Dans les écrits freudiens de 1911, le texte consacré aux Mémoires d'un névropathe du Président Schreber met en évidence, sinon l'importance d'une problématique sexuelle anale, du moins celle, quasi-équivalente dans le contexte qui nous occupe, de la mise en position passive au cours d'un rapport sexuel. En effet, rappelons qu'après avoir été nommé Président de la Cour d'Appel de Dresde, en juin 1893, le facteur décisif de la rechute de Schreber fut d'avoir été traversé, « un matin, dans un état intermédiaire entre le sommeil et la veille, [par] "l'idée que ce serait très beau d'être une femme subissant l'accouplement" » [1911c, p. ?]. Le Président Schreber entra donc dans le délire à partir de sa propre identification, vécue comme insupportable, à un être occupant, sur le plan sexuel, une position passive. Cette thématique devait rester le noyau de son délire. $\mathrm{Au}$ cours du développement ultérieur de la maladie, la position «féminine » tendit toutefois à être progressivement acceptée par celui-là même qui en faisait les frais. À défaut de pouvoir supprimer les sensations qui s'y trouvaient liées, et les sentiments d'humiliation qui leur étaient associés (Steiner, 2004), le Président Schreber se serait résolu de les changer en source d'une « béatitude femelle » qui parut le réconcilier avec son homosexualité latente.

Ajoutons cependant que, sur un autre versant, la question anale ressurgit de plus belle sous la plume de l'auteur des Mémoires d'un névropathe. Freud, en effet, souligne combien «Dieu», avec qui il considérait être en contact permanent, «soulève, chez Schreber, une indignation particulière par son comportement en ce qui concerne le besoin d'évacuer ou de ch... ». Et, de fait, il s'avère que, selon Schreber, Dieu «provoque par des miracles $[\ldots]$ le besoin d'évacuer les matières », car, du point de vue divin, « chi... est pour ainsi dire la chose ultime ». La jouissance sexuelle dont Schreber paraît frustré - puisqu'il n'y accède qu'en position passive - il se la réapproprie, sur le versant anal : "Celui qui est parvenu à se mettre en un rapport tel que le mien avec les rayons divins a pour ainsi dire le droit de chi... sur le monde entier. » $[\mathrm{REF}]$ (p. 225) 
Schreber, en « chi... sur le monde entier», se venge d'une position passive, subie. Il prend à son propre compte, non pas la volupté féminine (à laquelle il ne fait que se résigner), mais l'orgasme anal. Ainsi, il semble compenser la passivation subie sur un plan génital, de sorte à ce qu'un semblant d'équilibre s’établît.

\section{L'érotisme anal dans les textes d'Eduard Hitschmann, Johan van Ophuijsen et August Stärke}

Les thématiques citées plus haut furent réexaminées par certains successeurs de Freud, dans les années qui suivirent directement la publication du texte consacré aux Mémoires d'un névropathe. En 1913, Eduard Hitschmann, médecin et membre du premier cercle de psychanalystes viennois, publia dans l'Internationale Zeitschrift für Psychoanalyse un texte fort bref (3 pages) intitulé Paranoia, homosexualité et érotisme anal. Il y présentait l'histoire d'un jeune employé de banque, persuadé d'émettre des flatulences nauséabondes et se plaignant d'être devenu, par conséquent, l'objet des quolibets de ses collègues (ibid., .251) :

Il commença à se parfumer. Comme il pensait [aussi] avoir eu un rapport sexuel avec une prostituée sentant fort mauvais, il lui vint à l'esprit que là pouvait bien être l'origine de sa propre mauvaise odeur ; il la rechercha, pour constater, cette fois, qu'il ne lui trouvait plus cette mauvaise odeur. (ibid., p. 252)

Ce patient ne tarda pas à se sentir véritablement persécuté par ses collègues de bureau qui continuaient, disait-il, à se moquer des odeurs qu'il pensait émettre (252-253). Il courut de médecin en médecin pour expliquer ses maux, sans qu'aucun parvînt ni à le guérir, ni à mettre un terme à ses «hallucinations olfactives» (p. 253). Hitschmann, dans ses conclusions, liait ces phénomènes au refoulement de tendances homosexuelles et proposait de corréler la paranoïa à « l'érotisme anal » (p.254). Cette hypothèse lui semblait étayée aussi bien par l'intérêt montré par le jeune homme pour le fonctionnement de son propre système intestinal, que par son mélange d'attirance et de répulsion envers ses collègues de bureau.

Sept ans plus tard, dans l'International Journal of Psycho-Analysis, un autre auteur revint sur ces thématiques. Johan van Ophuijsen, médecin hollandais parti se former à Zürich, auprès d'Eugène Bleuler [référence], introduisit plusieurs considérations attenantes au sujet qui nous intéresse dans un écrit intitulé : Les origines du sentiment de persécution. «L'expérience m'a conduit à penser », écrivit-il, «que ce sentiment [de persécution] doit être en rapport au complexe anal» (p.235). Et de donner plusieurs exemples cliniques à l'appui de cette thèse.

Dans l'une des vignettes cliniques présentées par Ophuijsen, un jeune homme faisait état, à travers ses associations, d'une équivalence entre des loups, qu'il avait abattus en rêve, et la matière fécale qu'il «évacuait à travers ses boyaux» (p.237). Ophuijsen en concluait que «le "persécuteur" et le scybale sont tout simplement considérés comme des choses équivalentes ». Il rajoutait ensuite que des observations semblables, dont il ne doutait pas qu'elles eussent été faites, également, par ses collègues, permettaient de se demander si la «persécution anale » («anal persecution») n'était pas l'élément primordial à l'œuvre chez tous ceux qui souffrent du délire paranoïaque. (p.239)

Nous retrouvons la trace de ces questions et des débats auxquels elles donnèrent naissance jusque dans les années 1930, comme en témoigne, dans l'Internationale Zeitschrift für Psychoanalyse, la publication d'un texte intitulé : Le rôle des quantités anales 
et orales dans le délire de persécution et dans les systèmes de pensée analogues. L'auteur, August Stärke, était, comme Ophuijsen, un psychanalyste hollandais. Son hypothèse consistait à avancer que, dans la paranoïa, «l'excitation de la zone anale » (p. 6) rendrait patente une position homosexuelle jusqu'alors seulement latente car sublimée. Par là, il adviendrait une perte de l'équilibre psychique du sujet concerné. Elle le forcerait à remodeler complètement ses dynamiques libidinales, ce qui se traduirait tantôt par l'apparition, tantôt par l'aggravation, d'une pathologie délirante. Le réinvestissement, sur la personne propre du sujet, de la libido autrefois investie dans le monde, passerait donc la voie de l'érotisme anal, et de l'excitation qui l'accompagne (p.22).

\section{L'hypothèse bergerétienne d'une lutte contre l'angoisse de morcellement par pénétration anale}

Pour Jean Bergeret, dont les travaux furent publiés un demi-siècle environ après ceux de ses collègues dernièrement cités, la mère du paranoïaque est réputée phallique, narcissique, mais son image est occultée par celle d'un père qui serait, en fait, fantasmatiquement le grand-père maternel. Ces confusions identificatoires placent le sujet d'organisation paranoïaque dans une position difficile :

Il n'y a pas d'identification valable avec le parent de même sexe, demeuré tout puissant. Le caractère paranoïaque doit se défendre contre cet adversaire présumé, dont accepter l'amour serait considéré comme se soumettre à la castration phallique narcissique et à la dégradation homosexuelle passive. (Bergeret, 1974, p. 213)

Le choix homosexuel préférentiel de ces sujets serait, en fait, selon Jean Bergeret (1974) un choix intermédiaire et pour ainsi dire un passage entre le narcissisme, ou amour de soi-même, et l'amour objectal. Cet auteur s'appuie sur le schéma de Robert Fliess (1950) pour situer le niveau de développement du sujet paranoïaque entre le sous-stade anal de réjection et le sous-stade anal de rétention, qui lui-même s'inspire des travaux de Karl Abraham (1916).

Laurence Sagnes et Claude de Tychey (1989) ont mis à l'épreuve l'hypothèse bergerétienne théorique et différentielle selon laquelle l'angoisse de morcellement dans la paranoïa serait due « à une peur de la pénétration anale consécutive à l'orientation homosexuelle et à la fragilité du Moi corporel » (ibid., p. 91). Ce critère permettrait de distinguer la paranoïa des autres organisations au sein de la structure psychotique (mélancolie et schizophrénie). Toutefois, en accord avec les travaux du groupe de Lausanne (1985), Sagnes et de Tychey (ibid.) ont constaté qu'il n'y avait pas au Rorschach de manifestation franche de l'angoisse de morcellement et de destruction chez le sujet paranoïaque, probablement du fait d'une protection efficace associé à ses mécanismes défensifs rigides.

Cependant, en utilisant la méthodologie de Nina Rausch de Traubenberg associant les réponses du sujet et la symbolique des planches de Rorschach, Sagnes et de Tychey (1989) ont pu montré, planche par planche, la présence d'une thématique homosexuelle passive à travers les réponses cotées «perspectives anales », selon la proposition de Schafer (1954). L'angoisse réactivée face à la puissance phallique et le besoin de contrôler voire de supprimer tout référent phallique (aux Planches IV et VI du test à illustrer peut-être par un exemple de réponse), la faillite des assises narcissiques (aux Planches V et VII du test à illustrer peut-être par un exemple de réponse) et le fantasme de désintégration corporelle 
(Planche VIII du test à illustrer peut-être par un exemple de réponse) conduisent les auteurs à corroborer l'hypothèse selon laquelle l'angoisse de pénétration anale menace le sujet paranoïaque dans son intégrité identitaire, ce qui corrobore empiriquement la position de Bergeret. Récemment, d'autres cliniciens projectivistes ont confirmé l'importance de l'organisation sadique anale de la libido dans la paranoïa (Louët \& Azoulay, 2016).

Nous soulignons, par conséquent, combien il importe de repérer la présence de l'analité dans les protocoles : elle peut ainsi s'exprimer dans la critique de l'objet couleur ( «les couleurs sont sales, mal faites, peu nettes »), mais aussi à travers l'évocation d'images de saleté suscitant dégout et critique. Elles peuvent également être déplacées sur des représentations animales évoquant l'analité (cf. certains rongeurs, du type «taupe, rat») ou s'exprimer plus directement dans la référence anatomique : «l'anus, les fesses, le derrière, l'arrière-train ». Par extension, toute évocation de ce qui est caché, derrière les apparences, de ce qui se trouve derrière (dont nous faisons le parallèle avec la cotation «Perspective Anale » inspirée par Schaffer (1954)) « est marquée par des fixations anales et des angoisses de pénétration anale » comme l'indiquent Louët et Azoulay (2016)

Nous pouvons le constater: depuis la parution du texte de Freud consacré au Président Schreber et jusqu'à aujourd'hui, le rôle de l'érotisme anal dans le développement d'un délire paranoïaque a été au centre des attentions. L'utilisation clinique de ce signe a bien sûr évoluée en même temps que notre regard sur l'homosexualité (Lingiardi \& Capozzi, 2004), mais il semble important de ne pas céder aux pressions sociales, d'où notre retour rigoureux aux textes de Freud et ses collègues (Flanders et al., 2016), qui incitent à distinguer un processus psychique homoérotique d'un comportement sexuel véritable (Bergeret, 2002). Afin d'introduire, dans notre propos, un axe de lecture complémentaire, nous reviendrons, dans la dernière partie de cet article, sur l'apport théorique du psychiatre et psychanalyzste Karl Abraham. Ses travaux, en effet, le conduisirent à proposer des critères qui pourraient bien nous autoriser à distinguer, sur la base du lien à l'analité, la paranoïa de la schizophrénie. Mais, avant de nous avancer sur ce terrain, il nous a semblé plus pertinent de présenter le patient renommé par nous Manuel [suivi par le $2^{\mathrm{e}}$ auteur, psychologue et doctorante en psychologie].

\section{PRÉSENTATION DU CAS DE MANUEL}

Manuel est un jeune homme, plutôt grand, mince, à la peau mate, toujours soigné, élégant. Sa chemise blanche est repassée, il porte un pantalon de costume noir, des mocassins. Âgé de trente-trois ans, il a été hospitalisé une première fois, durant cinq semaines, à la demande d'un tiers (ses parents) il y a environ deux ans. À cette époque, Manuel n'était pas encore connu des services psychiatriques. Son internement apparaissait comme la conséquence d'une violente dispute avec ses parents, à leur domicile.

Comme sa mère, Manuel est informaticien. À ce sujet, il déclare : «Mon plaisir c'était l'informatique. J'ai toujours travaillé comme informaticien. Je fais de l'informatique depuis 4-5 ans. J'avais une machine à 30 000-40 000 euros dans ma chambre. » Il a exercé durant quelques années au sein d'une banque, au service «sécurité », dans un pays frontalier. Issu d'une famille relativement aisée, son mode de vie, avant le point de rupture 
que nous décrirons plus bas, semblait plutôt sobre, centré sur son travail. «Pour faire ce métier, il faut fonctionner comme un robot», déclare-t-il. Il vivait seul, sortait peu, sa vie sociale apparaissait quasi-inexistante. En revanche sa vie intellectuelle était riche. Pendant son temps libre, il aimait bricoler des objets, aller au cinéma, écumer les braderies à la recherche de cassettes de films anciens et d'autres supports de diffusion sur lesquels il écoutait des discours politiques, qu'il collectionnait. Ses cassettes occupaient une place très importante dans sa vie et il en reparle avec beaucoup de nostalgie, voire même avec «l'envie de pleurer ».

\section{À l'origine du délire, le traumatisme?}

Manuel a dû renoncer son emploi à la banque ainsi qu'à son appartement suite à un événement survenu en mai 2013, lequel a eu un effet dévastateur sur sa vie. Il pense avoir été cambriolé et violé durant son sommeil, et se sent, par conséquent, particulièrement humilié. Il nous dira : «Je suis une victime, j’ai perdu mon honneur ! J'ai perdu mon boulot et je suis parti avec une mauvaise réputation et en plus vous m'enfermez! C'est une injustice, un crime contre les droits de l'homme».

Quelques mois après la perte de son travail Manuel est retourné vivre chez ses parents, mais leurs relations se sont rapidement détériorées. Sa mère, d'après lui, contrôlait chacun de ses mouvements (vérifier les toilettes après son passage, la salle de bain, etc.). Cette dernière, originaire d'une famille algérienne émigrée, a initialement été mariée de force à un homme. Elle se serait, ensuite, sauvée avec son mari actuel, le père de Manuel, français d'origine allemande. Elle évoque la mémoire de son propre père avec un profond mépris, et nous a confié avoir souffert de la rupture avec sa famille : «Je pleurais tout le temps quand j'étais enceinte, j'en voulais beaucoup à mon père »

Devant son fils, lors d'un entretien familial, la mère de Manuel nous confiera aussi que, lorsqu'il «fait des crises »-qu'elle qualifie presque de diaboliques, car elle nourrit certaines idées au sujet de la spiritualité - son visage se transforme, et qu'elle y reconnait celui de son propre père. Il nous apparaît en réalité que cette mère a projeté à l'endroit de Manuel les caractéristiques négatives correspondantes à la personnalité de son père. À propos de son fils, elle nous dira en entretien : «Il fume, il boit comme mon père. » Comme pour coller au désir de sa mère, quelques semaines plus tard, Manuel, lors d'une énième dispute, imitera son grand-père paternel en s'adressant à son père en des termes injurieux.

Ajoutons enfin qu'à sa naissance, Manuel a subi une exsanguinotransfusion, car le rhésus de ses parents était incompatible. Il nous apparaît que ses souffrances somatiques périnatales pourraient avoir contribué à la fragilité de son développement narcissique.

\section{Difficultés du suivi hospitalier}

Les périodes d'hospitalisation sont vécues de manière très négative par Manuel. Il se révolte avec beaucoup d'énergie contre les conditions de vie, les règles du service, l'hygiène des autres résidents, et s'insurge contre son traitement antipsychotique depuis sa récente mise en place, quoiqu'il soit contraint de le prendre, ce qui démultiplie ses motifs de plainte. 
Lorsque nous écrivons ces lignes, Manuel attend sa future sortie. Il menace parfois de recourir à la force si nous ne le laissons pas s'en aller. Nous ne pouvons être qu'interpellés par la prégnance de l'analité dans son discours : «Je vais mettre des balles dans le cul à tous ces cons »; «Moi je me suis fait sodomiser, je suis une victime, j'ai envie de pleurer ».

Le transfert ambivalent que nous avions observé lors du premier entretien avec Manuel, il y a deux ans, nous semble donc atteindre son paroxysme. Néanmoins, il continue à se confier beaucoup, et, malgré son haut niveau de méfiance habituel, il semble nous considérer, la plupart du temps, comme son alliée. Dans la rencontre avec lui, nous devons être attentifs à ne pas nous laisser subjuguer par ses tentatives de séduction. Nous retrouvons en lui toute l'ambivalence qui organise sa relation à l'objet maternel. Cette mère incestuelle (Racamier, 2010) qu'il idolâtre, se distingue de celle qu'il haït par ailleurs, car elle a projeté en lui dangerosité et malveillance. À plusieurs occasions Manuel a rejeté notre fonction de psychologue, se drapant dans une position phallique pour rétorquer : «Non mais je veux bien aller boire un verre avec vous, je vous l'ai déjà dit, vous êtes bien mignonne, mais je n'ai pas besoin de psy! ».

Manuel n'en continue pas moins de nous «satisfaire » en se présentant comme convenu à nos rendez-vous ainsi qu'en adhérant à la psychothérapie. Il déploie tous les schémas de sa pathologie. En contrepartie, il nous contraint à rester son alliée, sans jamais remettre en question ses attitudes, ses propos. Lors des entretiens, il évoque ses théories, plus ou moins réalistes, au sujet du monde qui l'entoure. C'est en s'inspirant de faits scientifiques plausibles et s'étayant sur une culture générale relativement riche qu'il se maintient dans une tentative de recours à une pensée rationnelle. Mais en des points sensibles, de nets glissements, autrement dit, des pertes de liens à la réalité viennent assombrir le tableau. Par exemple, il nous a indiqué au cours de l'un de nos longs entretiens que nous serions tous des «poulpes» (certainement par référence au film de Matrix [Downing, 2007]). Nous vivrions dans une forme de logiciel où un ingénieur, une personne clairement identifiée, nous dirige et nous utilise à des fins que lui seul connaît, ou peut-être pour seulement se divertir, se jouer de nous.

Pour conclure ce versant de notre propos, ajoutons qu'il nous a semblé que, dans les jeux de corps, Manuel tentait de reproduire un certain mimétisme et, qu'au travers de cela, il tentait d'intégrer la fonction phallique que nous possédions - de son point de vue - au départ. Ces mouvements transférentiels nous donnaient l'impression d'un collage psychocorporel auquel nous ne pouvions nous soustraire. Cette forme de transfert, adressée à la fonction paternelle, et à forte charge érotique, nous fit penser plus tard, lors d'une supervision, que Manuel recherchait quelque chose de l'ordre de la pulsion homosexuelle. Nous avions remarqué d'ailleurs, lors d'entretiens où il exposait la plupart du temps des théories complotistes, qu'il faisait de nombreuses fautes d'accord (entre féminin et masculin). C'est à cette période, quelques semaines avant sa seconde hospitalisation, qu'il prit ses distances avec nous, comme s'il avait été comblé par cette transaction opérée fantasmatiquement.

\section{RETOUR SUR L'IDEE DE «VIOL HOMOSEXUEL »}




\section{Un point de cristallisation du délire}

Le point de rupture, dans l'histoire de Manuel, correspond, d'après ses propres dires, à un événement qui s'est produit en mai 2013. Lorsqu'il nous a relaté les faits pour la première fois voici deux ans, il s'est plaint, tout d'abord, que son appartement ait été cambriolé, une nuit, durant son sommeil. La porte blindée fermée à double tour ne laissait pourtant apparaître aucune trace d'effraction.

Selon Manuel, les cambrioleurs se seraient procurés le double des clés. Il se serait agi de personnes certainement entraînées, peut-être des espions ( «quelle genre de personne va enc*** quelqu'un?, des mecs qui ont fait l'armée, qui ont la légion d'honneur»; «faudrait regarder aussi du côté de ceux qui ont construit l'immeuble ») ou des personnes qui auraient entretenu des liens avec la banque dans laquelle il travaillait.

Manuel ne s'est donc pas aperçu de cette effraction dans l'immédiat, mais deux mois plus tard, en constatant que les cartons qui contenaient les vidéos qu'il collectionnait avaient disparus. Il nous a indiqué qu' « en y repensant bien », dès le lendemain matin des faits, il aurait éprouvé une forte douleur «au coccyx » et aurait remarqué l'apparition de troubles cognitifs («j'avais la tête en vrac»). C'est alors que Manuel aurait fait le rapprochement entre sa douleur au coccyx (qui a duré deux à trois jours) et cette disparition, concluant à un cambriolage, assorti très probablement d'un viol sans réelle certitude au départ. Les souvenirs du viol lui sont réapparus progressivement, sous forme de «flashs » nocturnes, ou réminiscences de cet événement traumatique. Manuel revoyait, pendant les mois qui ont suivi, très fréquemment, en pleine nuit, le visage d'un homme couvert d'un masque blanc. Cet homme le violait, dans ses souvenirs, ce qui lui aurait fait ressentir des sensations corporelles particulière (effets de transe, raideur, voire même paralysie transitoire).

Manuel justifie à présent ses pertes mnésiques, et son état de confusion antérieur, par l'utilisation d'un gaz puissant. Il nous a demandé, à plusieurs reprises, de nous renseigner sur les gaz disponibles sur le marché, destinés à effacer tout souvenir.

\section{L'élaboration ultérieure des idées délirantes}

Manuel a d'autant plus souffert de cet épisode que ses collègues de bureau lui auraient dit un matin, alors qu'il boîtait à cause de la douleur au coccyx, «mais tu es un

$\mathrm{PD} »$, en se moquant de lui. Il aurait ressenti, ce jour-là, une immense honte. Épuisé, il a donc décidé de prendre une année de congés, puis a démissionné.

Deux ans plus tard, Manuel continue d'élaborer des théories sur ce viol supposé avoir eu lieu voici deux ans. Il indique savoir, désormais, qu'il a été placé dans un camion citerne après avoir été exposé à un gaz pour le rendre amnésique. Il nous explique qu'il sait exactement comment est constitué un camion citerne de l'intérieur, alors qu'il n'est jamais entré, de son plein gré, dans ce type d'engin. Il revoit aussi, en images fixes, les sondes endoscopiques introduites dans les bouches d'aération, pour l'endormir grâce à du gaz. Actuellement, en entretien, il insiste sur le pouvoir de l'intestin et des spermatozoïdes et sur 
leur dangerosité en cas de contact. Il se remémore, par la même occasion, son viol, et allègue le souvenir d'avoir retrouvé un réservoir de préservatif déchiré. Selon sa théorie la plus récente, il aurait commencé à avoir des troubles suite au contact avec les spermatozoïdes de son violeur, qui se seraient logés dans son intestin, et dans ses parois, contaminant par là cet appareil «intelligent» qu'est le système digestif. Il nous dit souvent très sûr de lui, et en colère : «C'est la police que vous devriez appeler si vous voulez m'aider, je suis une victime, pas l'inverse. »

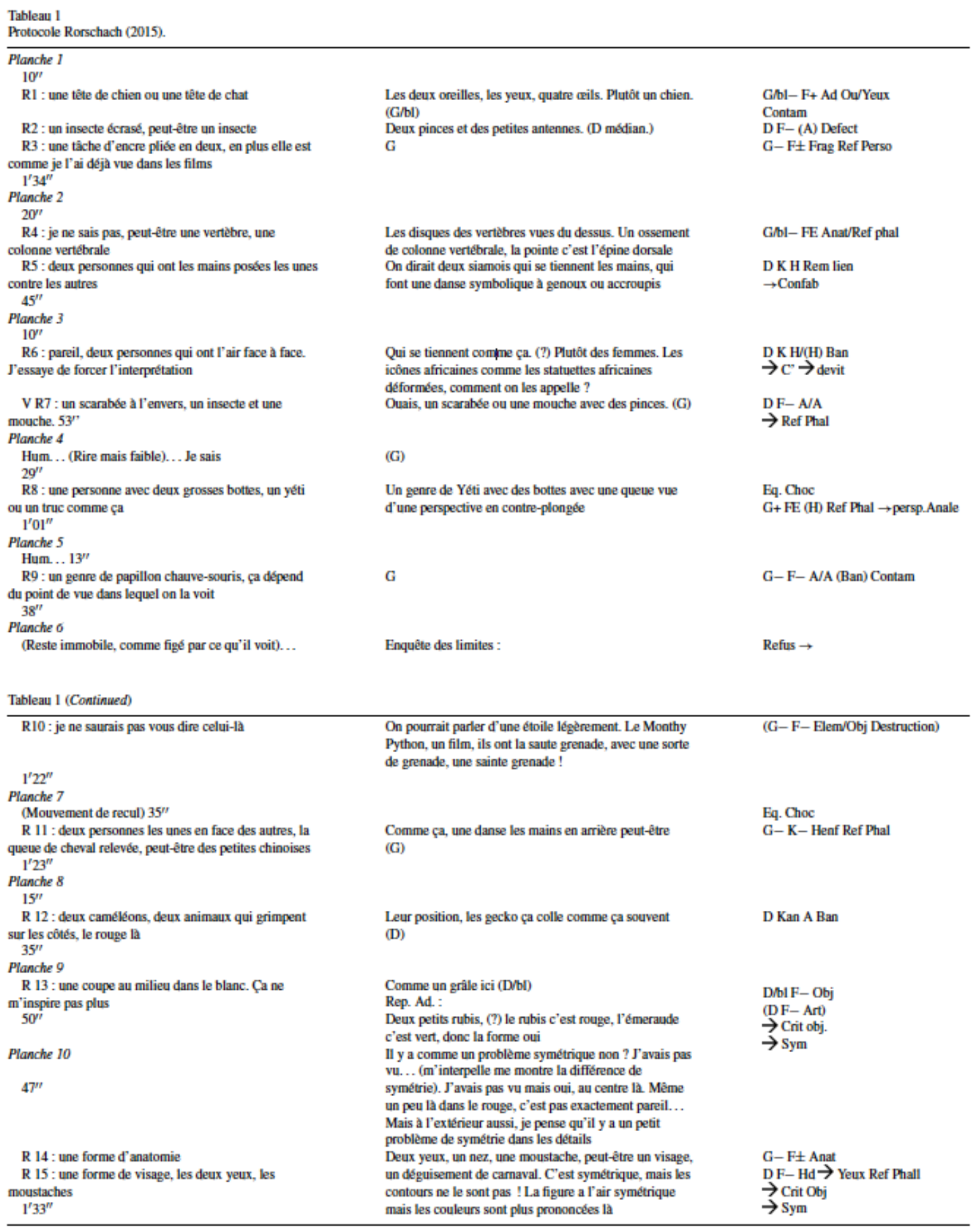


Nous appuierons présentement nos propos, avec pour support l'illustration de données recueillies dans le protocole du test de Rorschach de Manuel. Nous tenterons ainsi de mettre en lumière la dynamique intrapsychique du sujet afin d'en saisir plus finementson mode de fonctionnement.

\section{Analyse Rorschach}

\section{L'organisation défensive}

La projection est dominante dans le protocole de Manuel. La nature de la projection peut être parfois délirante comme nous l'observons à travers la réponse à la planche $2:$ «On dirait deux siamois qui se tiennent les mains, qui font une danse symbolique à genoux ou accroupis ». Cette réponse témoigne également de la dimension utile du délire comme une lutte contre la fusion à la mère. Nous observons aussi les signes d'une projection pulsionnelle agressive génératrice de désintégration de l'image du corps; par exempleà la planche $2:$ «Un insecte écrasé ». La projection peut-être être également de nature délirante à valence de destruction comme par exemple dans la réponse de Manuel à la planche 6 dans la réponse: «Le Monthy Python, un film, ils ont la saute grenade, avec une sorte de grenade, une sainte grenade !». Cette réponse illustre la manière dont l'environnement est vécu sur un mode menaçant et dangereux. La projection de la menace externe est associée à une recherche de position de toute-puissance («la sainte grenade »). Nous observons aussi comme dans tous les protocoles des sujets souffrant d'une psychose dissociée de notre échantillon - une projection renvoyant au morcellement de l'image du corps. Celleci infiltre tout le protocole et nous la mettons en lumière à travers des réponses telles qu'à la planche 9 : «Une forme d'anatomie ». La projection de nature paranoïde, incontournable au test dans ce type de personnalité aussi, est quant à elle perceptible à l'enquête, lorsque Manuel insiste sur la non-symétrie des planches. O. Husain (2005) remarque l'importance que les paranoïaques accordent à la symétrie au Rorschach, dans ses minuscules imperfections, comme si la symétrie constituait un questionnement en soi, un mystère impossible à élaborer. Ce besoin de prévisibilité, qui prend appui sur la symétrie représente un moyen de lutter contre l'imprévu au même titre que l'intérêt de Manuel pour les sciences, l'imprévu étant ce qui le soustrait au contrôle et favorise l'émergence du fantasme d'être piégé. Mais la projection de nature paranoïde est aussi repérable (quantitativement et qualitativement ) à travers les réponses «yeux » qui témoignent de la dangerosité de l'environnement et de la menace externe. La réponse «yeux» pourrait renvoyer à la culpabilité œdipienne, néanmoins dans ce contexte, associé à une contamination (qui correspond à un dérapage de l'imaginaire), (R1) nous optons en faveur d'une interprétation en terme de projection de la menace externe. Le sentiment d'être épié, menacé se retrouve dans la clinique puisque Manuel dort avec des couteaux sous son oreiller...

Nous saisissons enfin, grâce au test, la présence du dédoublement psychotique spécifique au psychoses dissociées. Manuel répond par exemple à la planche 3: «Deux personnes les unes en face des autres ». Ce dérapage langagier qui serait coté DV (verbalisation déviante) chez 
Exner, reflète un trouble de la pensée psychotique schizoparanoïde. DV chez Exner est la cotation attribuée à toute utilisation déformée d'un mot. C. Fleig et al. (1989) mettent cette cotation spéciale en rapport avec ce qu'ils nomment l' "écart sémantique » qui vient désigner dans le discours d'un sujet :

Toute modification de la convention qui unit dans le langage, le signifiant au signifié (...) Le DV vient donc souligner toute déviance par rapport à l'usage du signe linguistique. (Ibid., p. 844).

Cet écart sémantique au sens de C. Fleig et al. (1989) pourrait traduire, selon nous, l'état de fusion de Manuel à l'objet, générant une forme d'indifférenciation entre les objets, malgré sa lutte acharnée contre la dissolution identitaire.

À toute projection paranoïaque est associée le déni primaire comme l'a théorisé Freud (1911). Nous retrouvons les traces du déni en filigrane tout au long du protocole que ce soit à travers de fausses perceptions (voir du rouge dans du rose planche 8) ou à travers le déni de la relation (absence de kinesthésies interactives) ou encore à travers les réponses télescopées par absence de différenciation entre les objets (exemple R7 : « une mouche avec des pinces $\gg)$.

Des mécanismes secondarisés tels que le refoulement, le recours à la réalité ou l'intellectualisation sont parfois utilisés, mais rapidement mis en échec face à l'angoisse déclenchée par le contenu latent des planches. On peut voir que la représentation phallique paternelle $(\mathrm{Pl} .4)$ crée une angoisse forte (présence d'estompage associée à une équivalence choc) sans doute en lien avec l'orientation homosexuelle (lié à la présence de la cotation «perspect. anale » à cette planche).

\section{Le mode de relation à l'objet}

Dans la passation classique, rien ne nous indique que la relation d'objet est d'ordre persécuté/persécutant. Cependant, dans la passation psychanalytique, lorsque Manuel aborde sa relation aux femmes et à ses ex-petites amies, la jalousie est au premier plan. L'investigation du monde interne de Manuel via la passation associative met en lumière des relations vécues sous l'angle de la jalousie où le fantasme de trahison est prégnant. A la réponse «scarabée » à la planche 3, planche de la relation, Manuel associe : «Le symbole de la fertilité de l'Egypte. J'ai eu 5 ou 6 petites amies, plutôt sur la durée, plutôt constructif. [...] Il y a une copine qui a déconné, une rencontre avec un autre garçon. Si j'ai une copine qui va voir un autre garçon c'est la rupture... J'aurais bien aimé avoir un enfant ». Cet élément n'est sans rappeler le mécanisme de projection paranoïaque décrit par Lacan :

Le délire de la jalousie proprement paranoïaque est indéfiniment répétable, il rejaillit à tous les tournants de l'expérience, et peut impliquer à peu près tous les sujets qui viennent dans l'horizon, et même qui n'y viennent pas. (Lacan, 1981, p. 53) 
Le mécanisme de projection paranoïaque repose sur l'énoncé d'une tendance homosexuelle fondamentale mis en lumière par Freud (1911) dans le cas Schreber : moi (un homme), je l'aime lui (un homme). Il existe, avance Freud, trois façons de nier cette formule, d'où les trois types de délires paranoïaques. La première façon de nier l'assertion, celle qui correspond au délire de jalousie, serait de dire : ce n'est pas moi qui l'aime, c'est elle ma conjointe, mon double. Dans le délire de jalousie, on trouve au premier plan cette identification à l'autre avec interversion du signe de sexualisation. Si bien que le délire de jalousie est précisé comme l'une des formes du délire paranoïaque par Freud (1911c, p. ?)

\section{DISCUSSION : PARANOÏA ET SCHIROPHRENIE, AMBIVALENCE ET PRE- AMBIVALENCE}

La fixation anale pourrait ne pas être qu'un aspect contingent de certains délires paranoïaques tel que celui de Manuel, mais l'élément clinique permettant le diagnostic différentiel entre paranoïa et schizophrénie. C'est du moins ce que suggérait Karl Abraham dans un texte fondateur, l'Esquisse d'une histoire du développement de la libido basée sur la psychanalyse des troubles mentaux (1924). Il nous semble important de discuter la valeur sémiologique de ce signe clinique en confrontant les hypothèses d'Abraham à certaines données issues d'études de clinique projective.

Dans son texte, Abraham propose de mettre en relation ses propres observations cliniques de patients atteints de psychose avec l'hypothèse que leur processus de développement libidinal se serait trouvé figé, bloqué sur certaines phases. Il associe la paranoïa à la phase sadique anale primaire et, pour introduire son propos, commence par citer Van Ophuijsen et Stärke, les deux auteurs dont nous avons mentionné les contributions dans la première partie de cet article :

[Ils] découvrirent [...] que le «persécuteur » peut être ramené à la représentation inconsciente du scybale intestinal [= excréments] du patient. Ce scybale est inconsciemment identifié avec le pénis du «persécuteur », c'est-à-dire avec la personne du même sexe aimée à l'origine. [référence exacte]

Pour Abraham, il semble donc acquis que le paranoïaque met en équivalence ses excréments avec le pénis de son persécuteur. Ceci éclaire d'un jour nouveau les hallucinations de «viol homosexuel » qui assaillent Manuel. Semblable hypothèse nous permet également de mieux cerner le sentiment, dont témoignait notamment le Président Schreber, d'être mis en position passive dans un hypothétique rapport sexuel avec Dieu. L'objet anal, qui tient lieu de persécuteur, ne les lâche pas d'un pouce :

Pour le paranoïaque, l'objet d'amour est représenté par les fèces qu'il ne peut expulser. La partie de l'objet d'amour qu'il a introjectée se refuse à le quitter, de même que l'objet introjecté en totalité par le mélancolique exerce sur lui sa tyrannie. [référence exacte]

Contrairement aux patients atteints de psychose maniaco-dépressive, les sujets paranoïaques, précise Karl Abraham, ne mettraient en œuvre qu'une introjection partielle de l'objet d'amour. « Nous concevons », énonce l'auteur «que le mélancolique s'incorpore en 
totalité [cet objet] auquel il a renoncé, tandis que le paranoïaque n'en introjecte qu'une partie » [référence exacte]. Les effets d'une telle incorporation, pour partielle qu'elle soit, ne s'en font pas moins cruellement ressentir :

Dans la paranoïa, le persécuteur est [...] représenté par une partie de son corps que le patient ressent comme en lui : il voudrait se délivrer de ce corps étranger, mais n'est pas en mesure de le faire. [référence exact]

Les efforts que multiplie le patient paranoïaque pour refuser tout contact constructif avec autrui seraient donc à interpréter comme autant de tentatives pour jeter au loin un objet qu'il porte en lui-même. Le caractère désespéré de cette lutte l'entraîne dans une spirale où se succèdent, tantôt un découragement résigné, tantôt une reprise combative de la lutte.

Le caractère seulement partiel de l'introjection de l'objet d'amour (qui est, aussi, l'objet persécuteur) permettrait toutefois au paranoïaque de bénéficier d'une certaine marge de manœuvre dans ses tentatives de se débarrasser de lui. Les sujets paranoïaques témoignent bruyamment, envers cet objet, d'une ambivalence qui n'aurait eu le temps d'apparaître, selon Abraham, ni chez les patients atteints de psychoses maniaco-dépressives, ni chez les sujets schizophrènes.

Le paranoïaque n'échappe pas au conflit lié à l'ambivalence. Il espère [...] se défaire de la part incorporée et, conformément au niveau du développement psychosexuel où il se situe, ce n'est possible que par voie anale. [référence exacte]

Le projet de se défaire de l'objet persécutif reste voué à l'échec. S'il est livré à luimême, le sujet paranoïaque tendra, par conséquent, à répéter certains schémas de comportements agressifs. Leur libido ayant «régress[é] à la plus précoce des étapes sadiques-anales » [référence exacte], elle se traduit par des «mouvements haineux, hostiles, destructeurs de l'objet » [référence exacte] auxquels le clinicien a tôt fait de se heurter.

Dans les schizophrénies, la conflictualité du patient s'exprimerait, en revanche, de manière moins ouverte, en raison du fait que l'objet anal n'a pas encore été constitué comme tel. Toujours selon Abraham, le sujet schizophrène évoluerait dans un univers préambivalent, pré-anal, ce qui minimiserait ses expressions directes d'agressivité. Revers de la médaille, cette stase à la phase pré-ambivalentielle s'accompagnerait d'une tendance au repli sur soi. Le schizophrène s'oublie lui-même dans la symbiose avec l'objet oral (le sein). Il se confond avec l'activité même de "succion » [référence exacte] qui le maintient dans un flux perpétuel. Ayant affaire à l'objet sein, non-partiel, il se maintient plongé dans un monde où les objets, éminemment interchangeables, restent indistincts.

L'ambivalence associée ou non avec l'objet anal serait donc indicative de modes de structuration différents au sein des psychoses. La portée de ce signe semble échapper à certaines nosographies modernes qui éludent les facteurs psychodynamiques sous-jacents, pourtant révélateurs de tableaux cliniques distincts.

Ce propos gagne à être confronté à des résultats obtenus au Rorschach. Quatrevingts protocoles ont été recueillis en psychiatrie dans le cadre d'un travail de recherche différentiel (la thèse du deuxième auteur) avec des sujets diagnostiqués comme présentant des psychoses dissociatives. Cette démarche fera ultérieusement l'objet de notre part d'une analyse quantitative et comparative qui visera à différencier statistiquement les différents sous-groupes de notre échantillon de patients psychotiques dissociés (schizophrénie pure vs. schizophrénie paranoïde vs. paranoïa). Dans le tableau 2, nous présentons au lecteur un 
échantillon de réponses qui nous paraissent illustrer le contraste entre la fixation orale dans la problématique schizphrénique et la fixation anale dans la problématique paranoïaque.

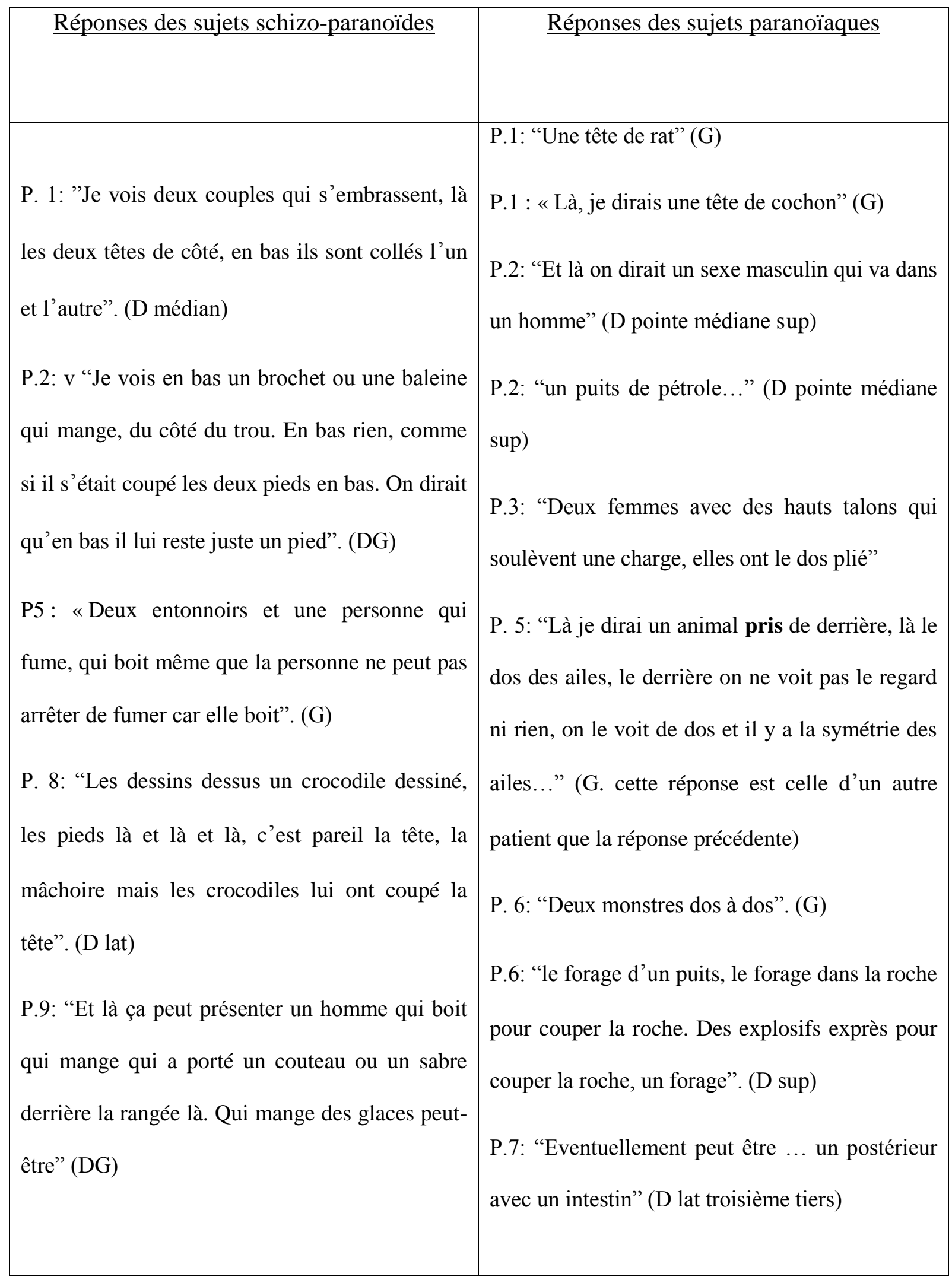




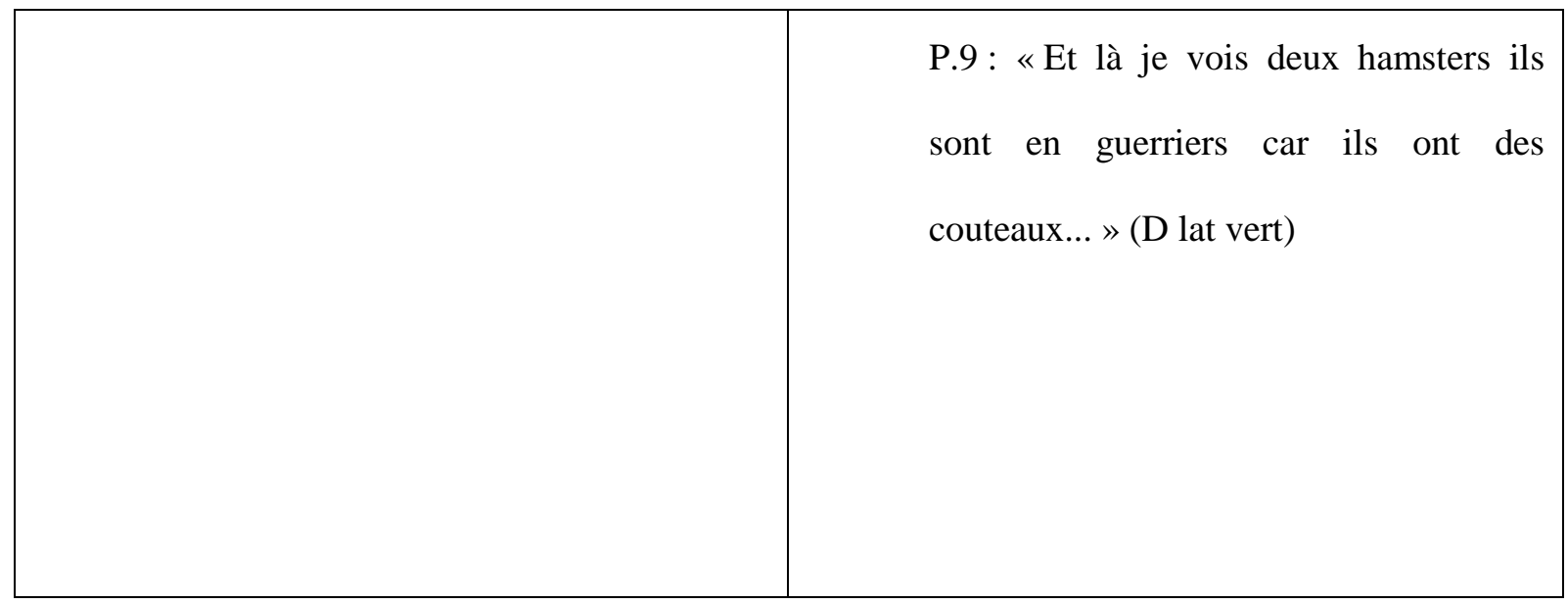

Tandis que les menaces de dévoration et de destruction envahissent la fantasmatique du sujet schizophrène, la menace de pénétration par voie anale signe la facture des protocoles paranoïaques.

Chez les sujets schizophrènes, la thématique orale (correspondant au stade oral primaire de Abraham) se reflète par l'usage de verbes tels que manger, boire, dévorer, s'embrasser, fumer. La thématique du cannibalisme renvoie également à la question de la succion du sein: Abraham, en effet, proposait de qualifier de "phase orale ou cannibalique » l'étape « la plus précoce » du développement de la libido, liée à la tétée [référence exacte]. La mention des «crocodiles »et de leur «mâchoire » nous semble renvoyer au même champ sémantique.

Chez les sujets paranoïaques, au contraire, la thématique anale ressort par la mention d'animaux classiquement associés à la saleté («cochons », « rats»), par la récurrence de l'idée de forage ( puits de pétrole») et, enfin, par l'insistance sur la thématique du «derrière » («dos à dos »). On note enfin la prévalence de l'image du sexe masculin, souvent associée à l'image d'un objet tranchant ou pointu (« couteaux », «talons »).

Le clinicien projectiviste pourra s'atteler, lors de l'analyse Rorschach, à différencier et repérer les contenus à valence sadique anale versus ceux à valence sadique orale dans une perspective différentielle qui enrichira la clinique des psychoses.

\section{CONCLUSION}

Cet article s'est donné pour but de faire le point sur la thématique de la fixation anale dans la paranoïa. Pour ce faire, il nous a semblé pertinent de partir aussi bien de l'étude des textes psychanalytiques consacrés à la question, que d'étudier le cas d'un patient, Manuel, suivi en psychiatrie depuis deux ans.

Il ressort des travaux de Freud et de ses successeurs que la fixation anale joue un rôle prévalent dans le développement d'un délire paranoïaque. Elle constitue, pour ainsi dire, le corrélat du refoulement de motions homosexuelles. Dans le propos et dans les écrits de patients diagnostiqués d'une paranoïa, ces deux thématiques apparaissent très souvent liées l'une à l'autre, l'homosexualité étant, par ailleurs, mise en équivalence avec la passivité au cours de l'acte sexuel.

Les propos du Président Schreber, dont le délire entier s'articule à une mise en position passive au cours d'un rapport sexuel imaginaire avec Dieu, font écho à ceux de 
Manuel, dont l'entrée dans la psychose fut marquée par une expérience délirante de «viol homosexuel ». Le protocole Rorschach de ce dernier témoigne de la prévalence d'un imaginaire anal. La fixation anale paraît également dénotée par son intérêt pour le fonctionnement du système digestif (présenté, parfois, sous forme de canalisations, ou tuyaux, dans lesquels circulerait un gaz asphyxiant).

Plutôt que de considérer le résultat de nos travaux comme un point d'arrivée, il nous paraît toutefois plus intéressant d'y voir un point de départ pour des investigations à venir. Il s'avèrerait, notamment, très intéressant d'approfondir les propositions d'Abraham quant à l'analyse la fixation anale dans le cadre d'une psychopathologie différentielle des psychoses. Mieux établir la distinction entre délires schizophréniques et délires paranoïaques passerait par une meilleure appréhension du fonctionnement, chez les patients schizophrènes, de la fixation au stade oral précoce qui implique un rapport à l'objet préambivalent. Tout un pan de la clinique psychanalytique et projective des psychoses reste donc à élaborer, à partir d'un héritage qui semble mettre en difficulté la psychiatrie contemporaine (ref. Prudent, Evrard, De Tychey, 2017).

\section{$\underline{\text { Références }}$}

[1] Prudent C, Evrard R, de Tychey C. La classification de la paranờa dans la psychiatrie américaine contemporaine : une revue de la littérature. Evol Psychiatr 2017;82(1):191-216.

[2] Bergeret J. La personnalité normale et pathologique. 2e ed. Paris: Dunod; 1974.

[3] McWilliams N. Psychoanalytic diagnosis: understanding personality structure in the clinical process. Silver Spring: Guilford Press; 2011.

[4] Freud S. Le Président Schreber : remarques psychanalytiques sur un cas de paranoîa (dementia paranoides) décrit sous forme autobiographique (1910). In: Bonaparte M, Loewenstein R, editors. Fuvres complête X. Paris: PUF; 1993. p. 227-304.

[5] Hitschmann E. Paranoia. Homosexualitat und Analerotik. Intemationale Zeitschrift fur Psychoanalyse $1913 ; 1(3): 251-4$

[6] van Ophuijsen JHW. On the Origin of the Feeling of Persecution. Int J Psychoanal 1920;4(3)-235-9. 
[7] Stärke A. Die Rolle der analen und oralen Quantităten im Verfolgungswahn und in analogen Systemgedanken. Internationale Zeitschrift fur Psychoanalyse 1935;21(1):5-22.

[8] American Psychiatric Association, DSM-5 task force. Diagnostic and Statistical Manual of Mental Disorders: DSM-5. Washington DC: American Psychiatric Association; 2013.

[9] Abraham K. Esquisse d'une histoire du développement de la libido basée sur la psychanalyse des troubles mentaux (1924). In: Barande I, Grin E, editors. Euvres complètes, 2. Paris: Payot; 1913-1925. p. 255-313 [traduction française 1966].

[10] Schifferdecker M, Peters UH. The origin of the concept of paranoia. Psychiatr Clin North Am 1995;18(2):231-49.

[11] Kendler KS. Kraepelin and the diagnostic concept of paranoia. Compr Psychiatry 1988;29(1):4-11.

[12] Macalpine I, Hunter RA. The Schreber case: a contribution to schizophrenia, hypochondria, and psychosomatic symptom-formation. Psychoanal Q 1953;22(3):328-71.

[13] Grotstein JS. The Schreber case revisited: schizophrenia as adisorder of self-regulation and of interactional regulation. Yale J Biol Med 1985;58(3):299-314.

[14] Dalzell TG. Freud's Schreber Between Psychiatry and Psychoanalysis: On Subjective Disposition to Psychosis. Londres: Karnac Books; 2011.

[15] Stroeken H, Johan van Ophuijsen. Padang/Indonesia 1882 - New York 1950: Luzif Amor 2009,22(44):7-44.

[16] Bergeret J. Homosexuality or homoeroticism? Narcissistic erostism. Int J Psychoanal 2002;83(2);351-2.

[17] Fejto K. L' homosexualité féminine primaire et secondaire dans la cure. Rev Fr Psychanal 2015;79(3):773-84.

[18] Flandersa S, Ladameb F, Carlsbergc A, Heymannsd P, Nazirie D, Panitzf D. On the subject of homosexuality: what Freud said. Int J Psychoanal 2016;97(3):933-50.

[19] Lingiandi V, Capozzi P. Psychoanalytic attitudes towards homosexuality: an empirical research. Int J Psychoanal. 2004;85(1):137-57.

20] Roughton R. Response to Beroent's "Homosexuality or homveroticisme narcissistic eroticism". Int J Psycnosh 2002;83(4):949-52 [Int J Psychoanal 83:351-62].

[21] Fliess R. Psychoanalytic reader. New York: UIP; 1950.

[22] Sagnes L, de Tychey C. Angoisse de morcellement par peur de la pénétration anale et paranoĩa : approche psychodynamique sur un cas à l'aide du test de Rorschach. Psychol Med 1989;21(1):91-5.

[23] Husain O, Merceron C, Rossel F. Psychopathologie et polysémie : études différentielles à travers le Rorschach et le TAT dépression, toxicomanie, simulation, plainte douloureuse, lombalgies, persécution, obsession, faux-self. Lausanne: Payot Lausanne; 2001.

[24] Schafer R. Psychanalytic interpretation in Rorschach testing. New York: Grune and Straton; 1954.

[25] Loult E, Azoulay C. Schizophrénie et paranoîa : étude psychanalytique en clinique projective. Paris: Dunod; 2016.

[26] Dollander M, de Tychey C. La santé psychologique de l'enfant. Fragilités et Prévention. Paris: Dunod; 2006.

[27] Racamier PC. L'inceste et l'incestuel. Paris: Dunod; 2010.

[28] Downing DL. Paranoiac visions and neo-realities in the recent cinema: reflections on Tausk's "influencing machine in schizophrenia". Psychoanal Rev 2007;94(6):991-1006.

[29] Husain O. From persecution to depression: a case of chronic depression. J Personal Assess 2015-97(3):230-40.

[30] Fleig C, Rebourg C. Six cotations spéciales du système synthétique d'Exner comme indicateurs de troubles de la pensée chez les schizophrènes paranoides. Psychol Med 1989;21(7):843-9.

[31] de Tychey C. Le Rorschach en clinique de la dépression adulte. Paris: Dunod; 2012.

[32] Lacan J. Le séminaire III. Les psychoses. Paris: Seuil; 1981. 